\title{
GALIÑEIRO, PASO DE LOBOS, NOVÍOS Y HÉROES. SOBRE EL EMPLAZAMIENTO DE LOS TÚMULOS DEL NW
}

\author{
por \\ JACOBO LEOPOLDO VAQUERO LASTRES*
}

\begin{abstract}
Resumen: Utilizando el conjunto tumular de Galiñeiro (situado en el límite entre las provincias de Coruña y Lugo, Galicia), se tratará de exponer como la vinculación de las construcciones a vías de tránsito naturales fue un factor de disposición uniforme y que, por lo tanto, debe ser tenido en cuenta como una característica inherente a la obra y a la búsqueda de visibilidad para la misma. Se comprobará que esta relación es más rica y compleja de lo que hasta el momento se creía, de modo que su consideración es un elemento imprescindible para estudiar el emplazamiento de túmulos en el NW peninsular.
\end{abstract}

Palabras clave: túmulos, megalitismo.

* Pintor Joaquín Vaamonde, 6-2 Dcha. A Coruña. Galicia (España). Departamento de Historia 1. Sección Prehistoria. Facultad de Geografía e Historia de la Universidad de Santiago de Compostela. Galicia (España).

"CUADERNOS DE ESTUDIOS GALLEGOS", Tomo XLI, Fascículo 106, Santiago 1993-94. 


\section{PREÁMBULO: TÚMULOS Y CAMINOS, UNA RELACIÓN ANTIGÜA}

Con frecuencia, los túmulos gallegos surgen en el campo asociados a una serie de caminos por los que discurrimos en su busca a veces sin demasiada convicción. Al lado de rutas que van desde corredoiras a autopistas, pasando por vías de tren, no es raro que con un simple "vistazo" detectemos la presencia de las tristes mámoas ${ }^{1}$.

Ya el intuitivo Federico Maciñeira, ejemplo señero de prospector, determinaba en su obra esa estrecha relación entre medoñas y caminos (Maciñeira y Pardo de Lama 1929; 1935; 1945; 1943; 1943-44). Esto es, además, una de las tradicionales observaciones de la más clásica arqueología gallega e incluso foránea (Valdemar Scmmidt 1906, Del Castillo, López Cuevillas, Manuel Losada, Barros Silvelo, etc.). En bibliografía más reciente, bien por alusión directa, bien a la vista de las representaciones gráficas adjuntas, se rastrea este hecho como el más generalizable en la distribución megalítica gallega ${ }^{2}$ (Vázquez Seijas 1953; Sobrino Lorenzo-Rouza 1953; Rodríguez Colmenero 1970; Filgueira Valverde \& García Alén 1977; Pérez Outeiriño 1.978; Criado Boado 1981; Patiño García \& García-Lastra Merino 1987; Patiño García 1985-86; Pombo Mosquera 1986; y un largo etc.).

Por otro lado para nosotros también resultaba clara la vinculación en las áreas que conocíamos bien del $\mathrm{NW}^{3}$. Sin embargo, la abundancia de este tipo de evidencias se contrarrestaba con un vacío total en cuanto a las interpretaciones. Optamos pues por partir de nuevo de la Serra de Loba y

\footnotetext{
${ }^{1}$ En numerosas ocasiones los que nos las ven o los que las desestiman son los constructores de carreteras, ampliadores de pistas, etc. Sin duda este es el motivo que, unido a las actividades post-concentración parcelaria y repoblación, destruye más yacimientos de este tipo en nuestra región.

${ }^{2}$ En el caso norportugués las referencias son igualmente abundantes (Jorge, V.O. 1984: 268-9).

${ }^{3}$ Serra da Loba, Cordal de Montouto, Serra do Bocelo (Criado Boado 1984), Cuenca de As Pontes (Grupo "Federico Maciñeira" A; Vaquero Lastres B) y Cha de Borneiro (Bello Diéguez 1988) donde en una prospección paralela a la excavación del monumento megalítico de Dombate se nos presentan unos terrenos donde la claridad de ese gran camino no aparecía y la localización de los yacimientos era muy complicada; sería donde comenzamos a aplicar y comprobar al mismo tiempo en el campo la validez de la hipótesis que vamos a detallar).
}

"CUADERNOS DE ESTUDIOS GALLEGOS", Tomo XLI, Fascículo 106, Santiago 1993-94. 
el Cordal de Montouto (Vaquero Lastres 1988) y, sin desligarnos de lo que el trabajo revisionista de $A$ Reborica (Vaquero Lastres 1989) nos indicaba, desarrollar ahora otro caso de estudio cuyas características son las idóneas para examinar este aspecto con una nueva perspectiva. El conjunto escogido es la necrópolis tumular ${ }^{4}$ de $A$ Serra das Medoñas de Galiñeiro. En ella se puede someter a otra revisión, creemos que nueva y muy rentable, el tema de los Megalitos y caminos.

La hipótesis que intentaremos mostrar a través de este trabajo es que esa vinculación aparece como un factor de emplazamiento uniforme pues es inherente a la propia esencia de la construcción, su visibilidad. Es conveniente que alguien vea lo hecho para ser visto. Por lo tanto, no es en absoluto descabellado el esperar que lo visualizado se relacione con los lugares donde se sitúan o por donde circulan los visualizadores.

Nos centraremos, por consiguiente, en los siguientes aspectos:

- Comprobación de como ese área definida como vía natural culturizada, vía de tránsito, va a ser un factor uniforme de explicación del emplazamiento.

- Acercarnos al emplazamiento como creador de monumentalidad desde otra perspectiva diferente (Reborica).

- Apertura hacia la búsqueda de la temporalización en la construcción del espacio en el caso del llamado megalitismo. La diferenciación entre construcciones dentro de un conjunto a la escala que sea (tanto de túmulos como rutas pues hablamos de bidireccionalidad), y en virtud de esto el acercamiento al movimiento de las sociedades en cuestión.

Nos situamos pues ante el reflejo material de dos ideas fundamentales en cualquier sociedad: la muerte y el movimiento; su consideración es la mínima reflexión necesaria para adentrarnos en lo que deseamos tratar en estas líneas.

\footnotetext{
${ }^{4}$ La utilización del término fue clarificada en Vaquero Lastres 1989; definimos con el a una agrupación de monumentos sin ninguna previa connotación cultural y temporal más allá que su consideración como túmulos.
}

"CUADERNOS DE ESTUdIOS GALLEGOS", Tomo XLI, Fascículo 106, Santiago 1993-94. 


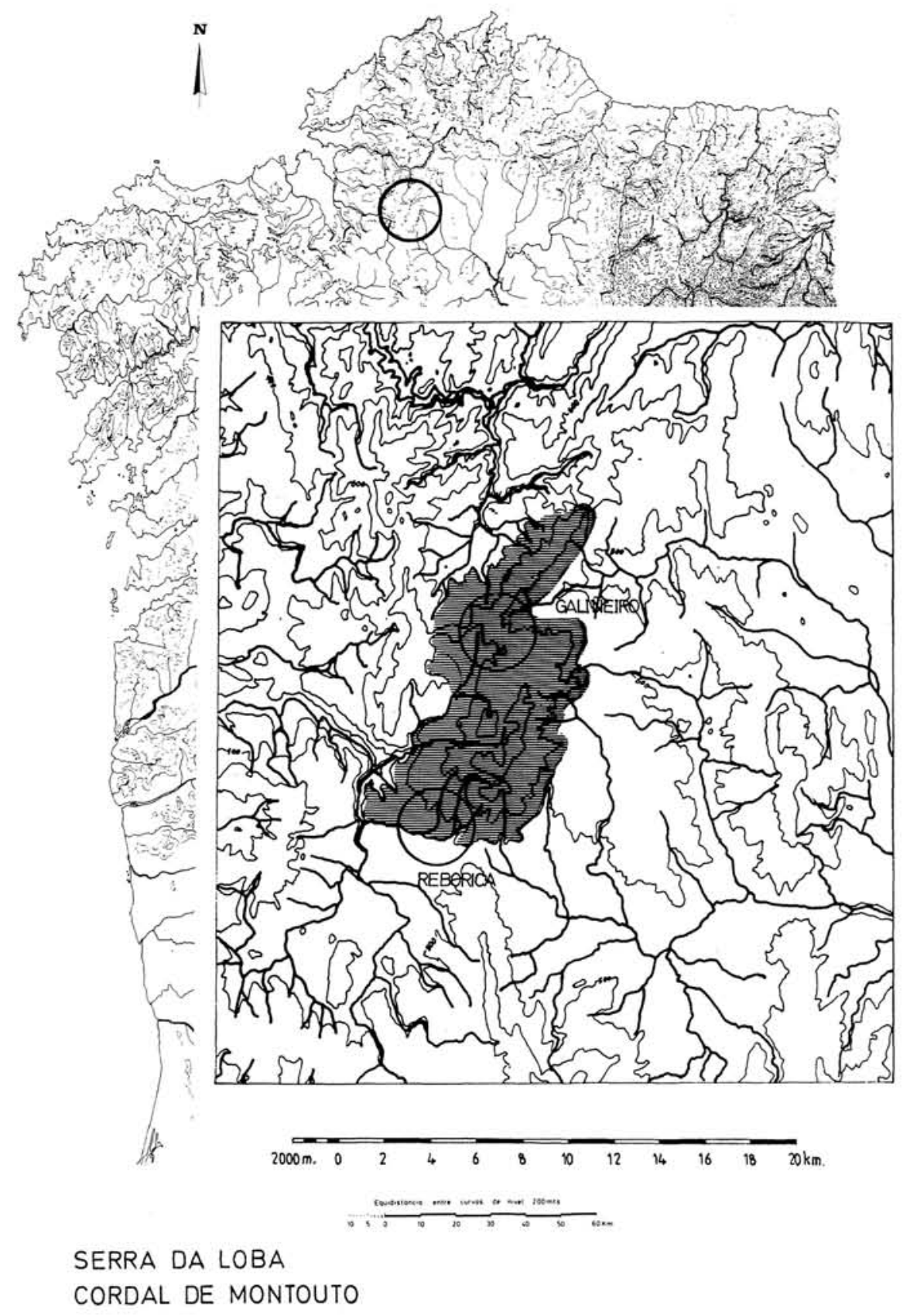




\section{1.- PLANTEAMIENTO: TÚMULOS Y CAMINOS, UNA RELA- CIÓN AMBIGÜA}

\subsection{Fuentes previas: primeros indicios}

Son varios los autores que desde las primeras fases culturales vinculan a los grupos humanos con movimientos más o menos constantes. Por otro lado, los ejemplos en el registro arqueológico europeo que nos hablan de una clara existencia material de caminos en etapas prehistóricas neo y calcolíticas son igualmente abundantes; su conservación es, en la mayoría de los casos, circunstancial y se relaciona con los mismos factores que motivaron su elaboración ${ }^{5}$.

En el NW peninsular se puede observar con cierta regularidad como, en esa abundante lista de referencias a la que aludimos, se nos suele hablar de caminos que se tienden a introducir en el pasado a través de una, en general erróneamente contextualizada, vinculación directa al emplazamiento de los monumentos; desde los solariegos campaniformes de As Pontes de García Rodríguez y sus "rutas comerciales" hacia el mar que nos detallaba Federico Maciñeira ${ }^{6}$ (1943) hasta la ilustrativa aproximación de Bello Diéguez, Criado Boado \& Vázquez Varela ${ }^{7}(1982 ; 1987)$. Estos investigadores recuperaban el tema demostrando que los apuntes anteriores no eran ni fruto de la imaginación de Maciñeira, ni se basaban en un caso excepcional. No parecen, sin embargo, buscar un análisis o una conclusión que tienda a ofrecer una explicación del hecho; a pesar de ello, si señalan la rentabilidad de la investigación.

De todas maneras, el problema de la bastedad de estas aproximaciones se pone de manifiesto si comenzamos una distinción de las evidencias.

Es bien sabida la profusión de caminos que se da en Galicia, no tan extraña si consideramos que estamos en una región con un paisaje antiguo y que además posee una gran dispersión del poblamiento; se entremezclan

\footnotetext{
${ }^{5}$ Por ejemplo, Coles (1990). En nuestra región tan sólo disponíamos de referencias dentro del folclore e interpretaciones de las mismas como la de Murguía que asimilaba la aparición de "maderos" en la remoción de una prototurbera a la posible existencia de palafitos relacionables con las medoñas de Buriz.

${ }^{6}$ El autor es el único que habla de un posible empedrado adscribible a la fase de construcción de los túmulos en la que denomina ruta de Bares (1943-38).

${ }^{7}$ En estos dos trabajos los investigadores ofrecen un detallado cuerpo de referencias tanto bibliográficas como inéditas en su momento, que vamos a aconsejar. Nos ahorramos su repetición y tan solo indicaremos aquellas no presentes en dichos trabajos.
} 
corredoiras, camiños vellos, grandes o de arrieiros, reales, pistas forestales, carreteras y autopistas; diferentes planteamientos de diferentes sociedades a la hora de discurrir por su espacio nos aparecen ahora en el que nosotros entendemos como un conjunto en muchas ocasiones de difícil interpretación. ¿Que ocurre si le acercamos nuestros, a veces, no menos masificados túmulos en busca (ahora si) de sociedades específicas?.

No nos equivocaríamos si dijesemos que la inmensa mayoría, sino la totalidad, de los túmulos conocidos en el NW ibérico los podríamos poner en relación con un camino del tipo que sea; desde los de As Pontes y Bocelo y su Camiño Real hasta los de la carretera nacional N-VI en la zona limítrofe entre Lugo y Coruña. Si esa relación fuese presentada sin más como un dato, no estaríamos aprovechando sin duda la connotación subjetiva de la palabra, manipulando en definitiva nuestra observación. Es decir, presentaríamos una vez más lo que es obvio.

En efecto, es necesario intentar romper esa confusa unidad funcional, tan falaz como considerar el conjunto atemporal de tumbas dentro de la comarca.

En todo ello tan solo observamos factible la siguiente apreciación que ejemplificamos en el todopoderoso camiño real: en numerosas ocasiones el lugar escogido presenta interés tanto para los "paseos" de los modernos gallegos como para obras de los constructores de túmulos.

¿Se da también en algún caso unidad de condicionantes? ¿Ese condicionante es uniforme como factor de emplazamiento del túmulo? ¿No podemos acaso matizarlo tanto en el caso de las construcciones como en el de los propios caminos?. Pretendo salir de ese nivel de obviedad en el que se mueve el tema.

El siguiente paso es, por lo tanto aclarar el significado del manoseado camino; fijemos pues la atención en la palabra camino, en el concepto que representa.

Si consideramos una definición general se trataría de una franja de terreno para transitar construida expresamente o formada por el uso; surge pues una connotación física independiente de lo que es el movimiento en sí y que hace depender la aparición de ese camino de circunstancias variadas que pueden ir desde las meramente ambientales hasta las conductuales, en el caso animal, y/o culturales, en el caso humano.

Baste por ahora aclarar que, partiendo de ese camino como alteración antrópica del medio, su aparición siempre tendrá un carácter cultural. Hay una diferencia importante entre lo que es la formación por el uso y la construcción: es fácil adivinar que en el primero de los casos las causas 
son de tal variedad que el hacer estimaciones sobre si se da o no quizás no fuese rentable; un movimiento periódico quedaría reflejado en la formación de un camino, pero, relacionado con lo anterior, la construcción de un camino supone una intencionalidad que entra a formar parte de una nueva consideración del movimiento, un tratamiento concreto de éste que ya no responde únicamente al paso del tiempo. En este sentido vemos como alteraciones puntuales del terreno se tienden a relacionar con el primero de los procesos, distinto al segundo, quizás ejemplificado por las vías en la antiguedad ${ }^{8}$.

A raíz de esto y para concluir, nos encontramos ante una acepción más restringida que definiría al camino como la ruta elegida para ir a un lugar determinado, desposeyéndolo entonces dicha definición, tanto de una formación como una construcción física.

\subsection{Método alternativo}

Lo que llevamos expuesto nos indica como en el caso gallego (y a veces no solo en él) siempre se ha planteado la relación camino (físico)megalitos. Para nosotros esto se manifiesta, como hemos intentado explicar, impreciso y desde luego no como un criterio generalizable, aunque puede darse, en el mundo denominado megalítico.

Vamos a procurar llegar un poco más allá proponiendo la vinculación alternativa: vía de tránsito-túmulo.

La diferencia con posicionamientos anteriores es esencial: se asocia el carácter de monumento de la obra tumular con un área de discurso, tránsito por un espacio; esta concepción presenta las siguientes ventajas claras:

- Elimina la connotación física con la que suele emplearse la palabra camino, connotación necesaria en el caso de obras públicas de una sociedad estatal como la romana pero de muy dudosa extrapolación a comunidades agricultoras como las que podrían haber levantado parte de las medoñas gallegas.

- Permite trabajar con un concepto atemporal (al igual que hacemos con la monumentalidad de los túmulos), trabajar exclusivamente con es-

\footnotetext{
${ }^{8}$ Conviene recordar a propósito de la cita, que otra de las evidencias constatadas con frecuencia son la relación existente entre enterramientos con esos caminos construidos, si bien esto se da en esos tiempos y sociedades posteriores cuyo caso más popular sea la propia Roma.
}

"CUADERNOS DE ESTUdiOS GALLEGOS", Tomo XLI, Fascículo 106, Santiago 1993-94. 
pacios, organización y modificaciones del mismo ${ }^{9}$, e introducir el tiempo oportunamente, cuando la certeza de la rentabilidad de su utilización sea la suficiente, esto es:

- Disponemos de un concepto operativo que nos va a permitir acercarnos a la culturización de la naturaleza, la transformación de los diferentes espacios culturales a través del paso de la vía "natural" a la vía cultural. Es ésta la que puede ser ejemplo o no de una modificación pretendida y visible de la naturaleza y en ese punto, o antes entrar en relación con otra clara modificación como es el levantamiento de un túmulo. ¿Es el túmulo una primera modificación en relación con una vía "natural" culturizada o es una modificación más en relación directa a otra previa, el camino construido?, nos parece un buen punto de partida.

\section{2.- DATOS: TIERRAS Y MEDOÑAS, UNA GEOGRAFÍA TUMULAR}

Hemos encontrado en la prospección de Serra da Loba y Cordal de Montouto una base idónea sobre la que trabajar, la necrópolis de la Serra das Medoñas y Galiñeiro, conjunto tumular de 21 construcciones en una extensión menor de $1 \mathrm{~km}$. cuadrado*. ¿Estamos verdaderamente ante un grupo cultural unitario?, ¿son los mismos los condicionantes? y, sobre todo, ¿por qué esa concreción de monumentos? La profundización en los aspectos arriba descritos quizás aclaren alguna de estas cuestiones.

Dentro de lo que podemos tratar como un relieve montañoso antiguo poco accidentado, el grupo se extiende sobre una pequeña penillanura terminal con pendiente suave SE. Las únicas referencias antiguas significativas sobre los yacimientos son las de los trabajos de Luengo Martínez (1952) y las anotaciones de Hernández San Pelayo (1922-42). Por otro lado, en Vaquero Lastres (1987) se ofrece una descripción pormenorizada de cada uno de los túmulos.

La diversidad de las características de estas medoñas es significativa en cualquiera de las dialécticas que analicemos (Criado Boado \& Fábregas

\footnotetext{
${ }^{9}$ En los párrafos siguientes plantearemos como múltiples caminos (ej. pista forestal, camiño real, etc.) que se pueden materializar en una única vía de tránsito (ej. cordal).

* Años después he comprobado la existencia de uno pequeño al lado de M40 y la posibilidad de otros en el área de afloraciones graníticas.
}

"CUADERNOS DE eStudios GALlegOS", Tomo XLI, Fascículo 106, Santiago 1993-94. 


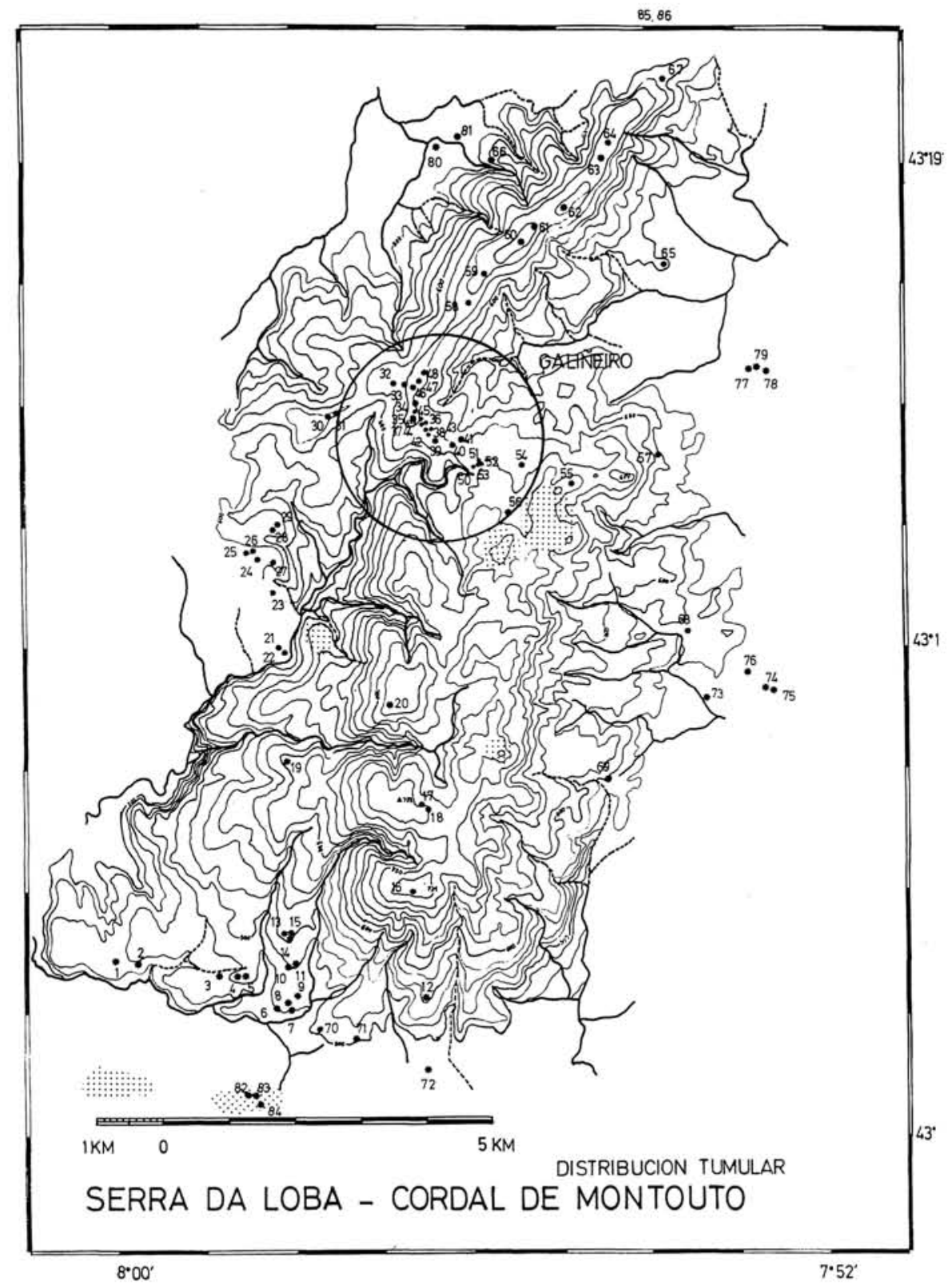




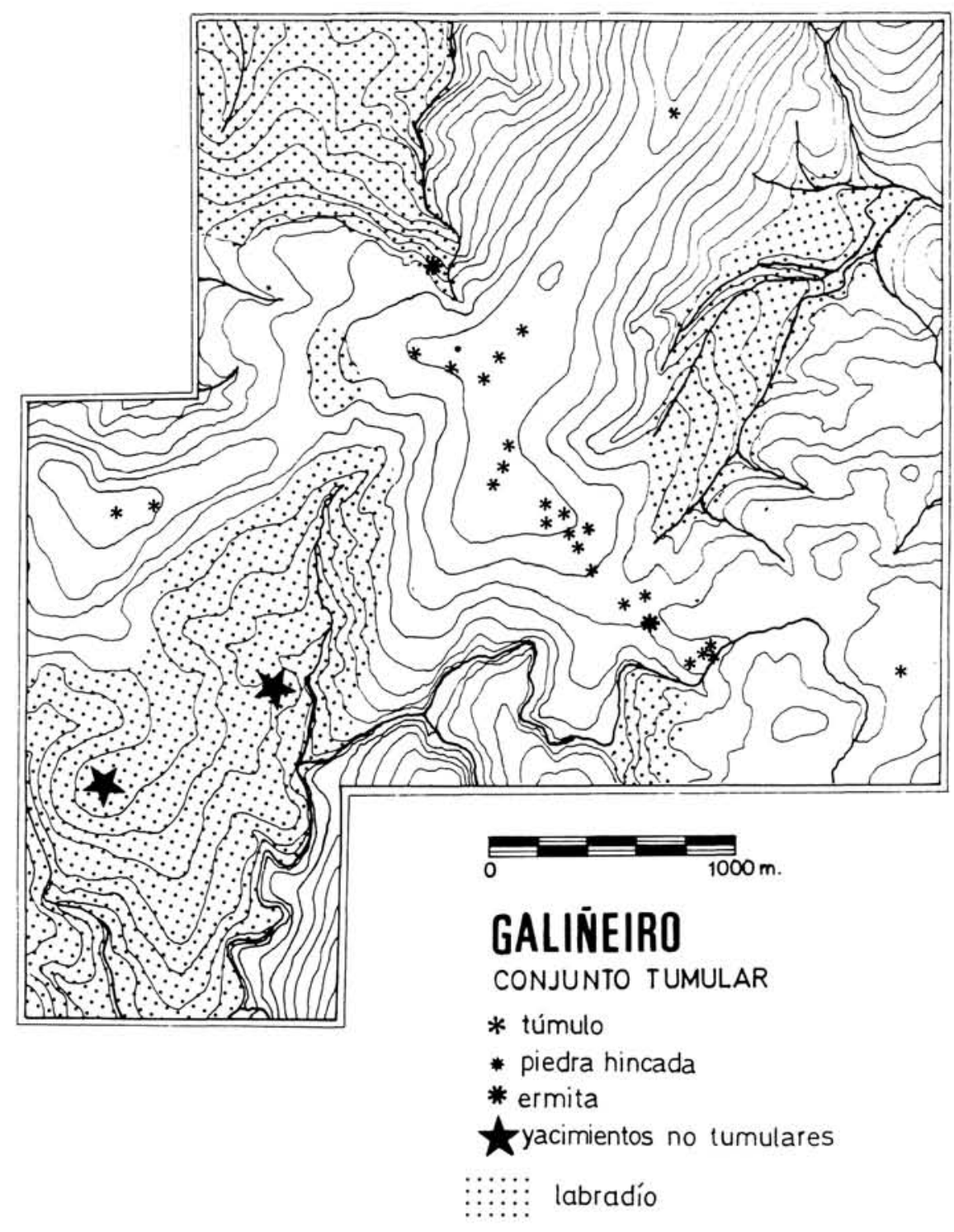


Valcarce 1989a). Arquitecturas tumulares de dimensiones que van desde los 30 m. de diámetro y 2 de altura (aprox. en M 32 y M 40) hasta las reducidas bien representadas por M 52 con $13 \mathrm{~m}$. de diam. y poco más de 0'50 de altura. Las estructuras interiores parecen ser igualmente diversas; desde cámaras cerradas como la que nos describe Luengo en M 40 o los violadores de M 41 hasta la bien diferente presumible la citada M 53. Por último, la distribución también resulta interesante; podemos observar como los túmulos de mayores dimensiones enmarcan el conjunto de la línea de pendiente hacia el SE, de tal forma que se aisla un grupo que es el normalmente presentado por las referencias, tanto bibliográficas como etnográficas (se suele hablar de 12/13 o en todo caso de 14 medoñas). Al $\mathrm{N}$ la conexión con la cuerda de A Loba se lleva a cabo a través de M 46, 47 y 48 desde donde se goza de una perfecta visión de dicho grupo. Por último la pequeña agrupación hasta el momento desconocida de M 50-53 aislada al S al lado del arroyo del Barranco da Loba.

Geológicamente es una zona de contacto entre la mancha de granito del Cordal de Montouto, granodiorita y una potente banda esquistosa, a ambos lados de la cual se disponen unas finas líneas de cuarcitas.

Las aldeas y casales campesinos más cercanos son las de Galiñeiro en la provincia de Lugo y la de Os Cainzos del lado coruñés. La parte occidental de la pista que discurre por el medio de la penillanura sirve de límite provincial. Además, el propio centro de la cruz topográfica significa, a través de una pedra fita ${ }^{10}$, la unión de 3 municipios (Monfero y Aranga de Coruña; Guitiriz de Lugo) y de otras tantas parroquias. El mojón se enclava en el cruce del camiño real de Betanzos a Vilalba que viene por la cima de A Loba desde el W, y el citado anteriormente en sentido SE.

Por último decir que, al igual que numerosos yacimientos gallegos, ha sido llevada a cabo una mas o menos efectiva cristianización, de manera que la necrópolis se encuentra enmarcada por 2 capillas de construcción tradicional, la de la Asunción al $\mathrm{N}$ y la de San Vitorio al S, al lado de la cual se celebra una romería, así como por dos cruceiros, el de Carballiño y el propio de San Vitorio.

${ }^{10}$ Piedra hincada en el cruce de caminos al lado del Cruceiro do Carballiño (cruz tradicional gallega) en la zona conocida como Cruceiro da Loba.

"CUADERNOS DE ESTUDIOS GALlEGOS", Tomo XLI, Fascículo 106, Santiago 1993-94. 

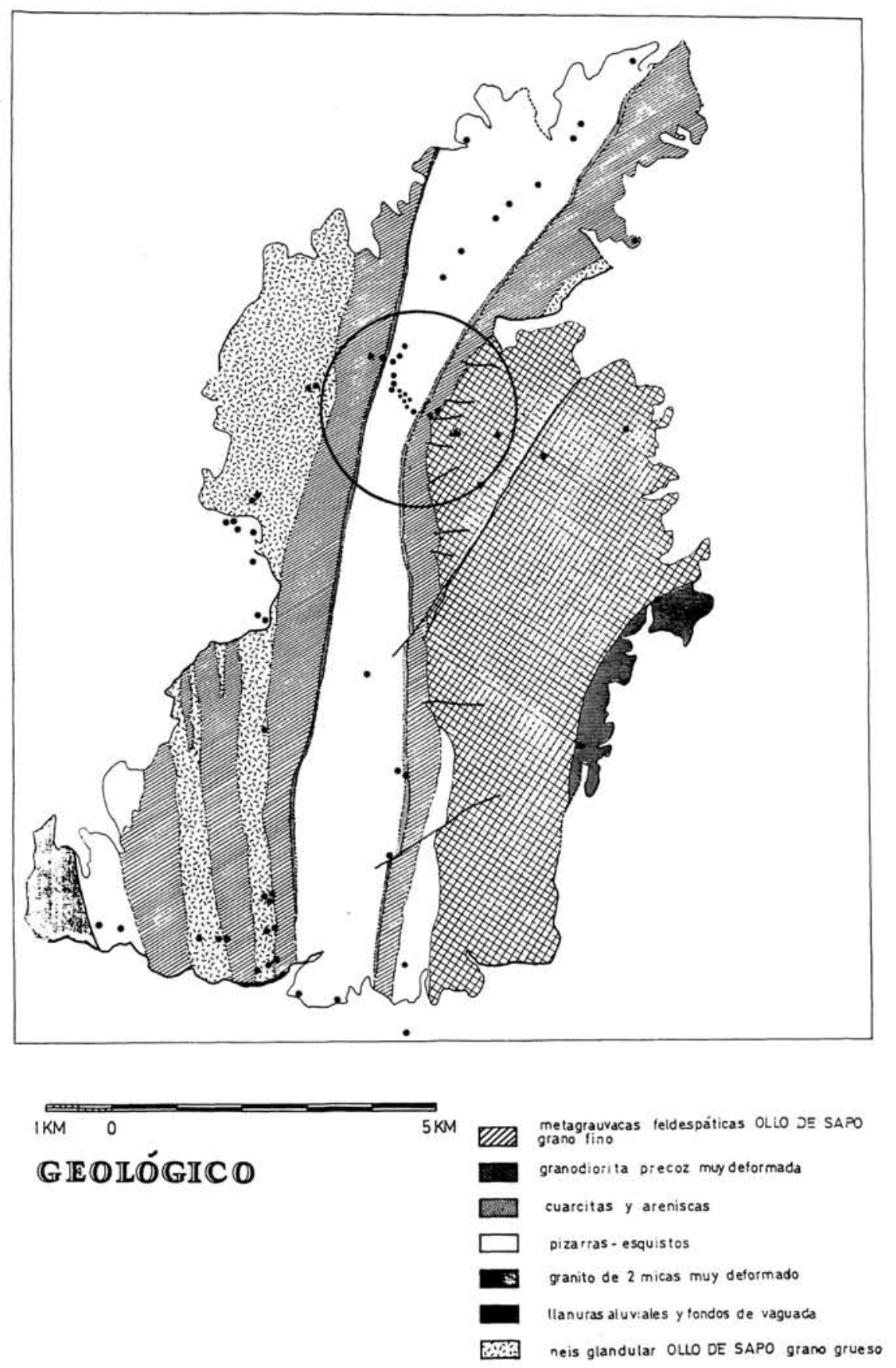


\section{3.- ANÁLISIS DE LOS DATOS}

\subsection{Primer nivel de análisis: geografía del tránsito por Galiñeiro}

A continuación, como estrategia metodológica, vamos a intentar describir esquemáticamente el tránsito en una región de forma que podamos diferenciar esas vías "naturales"11, distinguibles por su aprovechamiento, por los tipos de discurso por el terreno a los que se asocian. No olvidemos que ese tránsito es un todo y que nuestra simplificación solo busca una rentabilidad en la explicación.

En nuestro caso existen dos tipos de terrenos. Por un lado encontramos lo que podemos tratar como tierras altas que ocupan las sierras propiamente dichas, las penillanuras terminales, cordones y buena parte de las dorsales de estribación, en general dedicados a monte, pasto natural o repoblación desde que se abandonó la práctica de las rozas; y por otro lo que se puede definir como tierras bajas, con lo que denominaríamos los valles interiores de la montaña, normalmente cultivados y habitados.

En cada una de estas zonas nos encontramos una serie de claves de desplazamiento, es decir, las claves que nos permiten el acceso a todo el terreno y que se basan en un conocimiento exhaustivo del mismo. ¿Por dónde cruzaremos el río? ¿Por dónde subiremos a la cima de la montaña? y lo más importante ¿Por dónde la atravesaremos con mayor facilidad?, son todas preguntas ineludibles que han de hacerse al plantearse el movimiento.

Teniendo en cuenta las condiciones geográficas, podemos aislar los tipos de vías de tránsito y las áreas de paso a lo largo de la zona de trabajo:

a) Tránsito a lo largo de las tierras altas. Sin duda lo que más fácilmente podemos reconocer es la figura de la corda, lo cual podría ser válido ahora pues estamos ante un caso típico (Serra da Loba).

En virtud de ella proponemos y destacamos la utilidad del concepto de cruz, presente por necesidad en el relieve montañoso. Se dan dos casos (que en realidad se establecen sobre una diferencia de matiz) en base a una característica común: punto de encuentro de dos vías. Punto de intersección entre dos cuerdas o más de igual entidad (caso de Cruceiro da Loba; quizás sea esta la concepción tradicional), o entre una y sus dorsa-

\footnotetext{
"Venimos manteniendo, si bien con cierta grima, la imprecisión al uso de este adjetivo, pues al definir esa vía esperamos que dicho calificativo sea reconsiderado.
}

"CUADERNOS DE ESTUdiOS GALLEGOS", Tomo XLI, Fascículo 106, Santiago 1993-94. 
les de estribación de acceso. No hace falta aclarar que el término implica la consideración de las figuras tipo cordal como zonas de desplazamiento, pues estamos hablando del acceso a las mismas.

b) Tránsito a lo largo de las tierras bajas. Los problemas de desplazamiento generalizables son los planteados por las corrientes de agua (barrancos, cauces anchos, fuerte caudal, etc.). Se han de buscar para el paso los lugares en los que arroyos y ríos ofrezcan mayores facilidades. En Galicia la solución es el concepto de PORTO/VAO, que es así como se denomina (entre otros apelativos) el lugar utilizado para cruzar la corriente.

c) Tránsito desde las tierras altas a las bajas. El acceso a las rutas altas se efectúa a través de las dorsales de estribación de la propia sierra en cuestión, de manera que unas van a ser más propicias que otras para la penetración. Las partes bajas de estas dorsales pueden y suelen estar relacionadas con portos de los arroyos que las cierran en las zonas bajas; mientras que en las zonas altas ya dijimos que podemos incluir la intersección en una amplia definición de cruz.

d) Tránsito entre las tierras bajas. En la escala que tratamos es la figura del collado la que va a utilizarse prioritariamente en el paso de una zona baja a otra cuando estas se encuentran separadas por un accidente montañoso.

\subsection{Segundo nivel de análisis: etología, etnología y mitología del tránsito por Galiñeiro}

En este apartado vamos a tratar de presentar el área de Galiñeiro como una zona de discurso, una vía de tránsito especialmente caracterizada, abordándolo desde dos enfoques complementarios.

La zona de trabajo ha sido usada como vía de paso, comunicación en muy diferentes épocas y para muy diversas intenciones. Por ella se han movido mouros, navios, lobos, reyes, romanos, campesinos, héroes, franceses, caballos, investigadores y muertos. Organizando toda esta "base documental", nos podemos ceñir a tres ámbitos fundamentales que definirían esta zona como área de paso. 


\section{La evidencia etológica}

En primer lugar una sistemática observación de la naturaleza, constataciones etológicas sobre el movimiento animal ${ }^{12}$.

Una aproximación basada también en encuestas a los vecinos de la zona nos indica la utilización del terreno por el ganado equino y vacuno:

- Paso del cordal de A Loba, que habitualmente recorren, a las penillanuras elevadas de Montouto.

- Circulación hacia las partes más bajas de pasto de monte a ambos lados W y E de la Serra.

- Evidente empleo del pequeño paso, también aprovechado como abrevadero, del nacimiento del Barranco da Loba.

De igual manera la zona era conocida por los paisanos por ser el lugar por donde los lobos ${ }^{13}$ pasaban de una sierra a otra antes de que su especie cayera en desgracia.

\section{La evidencia etnográfica}

La observación de las comunidades rurales actuales de la región, su movimiento y la organización de su espacio resulta igualmente orientativa.

La pista de Cainzos (reformada y ampliada de modo periódico) y su cruce hacia Lugo aparece atípicamente frecuentada teniendo en cuenta las características poblacionales de los alrededores ${ }^{14}$.

La Serra das Medoñas de Galiñeiro es utilizada por los campesinos como paso entre las zonas de valle de Lugo (Guitiriz) y Coruña (Aranga). En ella es posible encontrarse desde un vecino de Galiñeiro que va a Leiras paseando después del almuerzo, hasta la juventud que el sábado de noche recorre los bares de uno y otro lado de las sierras de la dorsal meridiana gallega. Las personas de mayor edad nos definieron varias veces los terrenos como el lugar por donde, cuando eran jóvenes, cruzaban la provincia para "ir ás mozas".

\footnotetext{
${ }^{12}$ Una introducción para su especificación se puede consultar en Lorenz 1986; Eibl Eibesfeldt 1983 y Alsina 1986).

${ }^{13}$ Este animal debió ser ciertamente abundante sobre todo en la amplia extensión del Cordal de Montouto donde aún hoy pudimos ver alguno. Por otro lado el movimiento de lobos pudo también ser constatado en 1990 durante la campaña de excavaciones de Forno dos Mouros en la Serra do Bocelo.

${ }^{14}$ Una densidad baja en terrenos de montaña poco productivos dedicados a la ganadería semi salvaje y una agricultura de autoconsumo en las zonas deprimidas. Las zonas que se realizaban en la montaña hace 50 años han dejado de hacerse (Catastro del Marqués de la Ensenada).
} 
Todo esto se ve reflejado en el conocimiento exclusivo que en una superficie de muchos kilómetros cuadrados se tiene del collado de Galiñeiro como tal.

Es clara la importancia que tiene para el movimiento campesino el conocer terrenos de estas características en virtud de los medios de desplazamiento de los que disponen. En la región que presentamos, al igual que en todo el NW, esto queda de manifiesto si nos detenemos brevemente en el análisis de la toponomía menor: podemos comprobar como se detallan a la perfección en las denominaciones campesinas (Porto Naveira, Portorrosa, Cruz do Bruño, Portoluz, etc.) muchas ya olvidadas y otras carentes de sentido en la actualidad (As Balsas, Porto Os Carros, etc.). La Serra das Medoñas de Galiñeiro nos ofrece varios ejemplos como Cruceiro da Loba o Barranco da Loba. Es significativo el comprobar como en la región se denominan los regatos con el nombre de su porto (arroyo de Porta Lameira, de Pontorrosa, etc.).

\section{La evidencia mítica}

Además de los consabidos mouros, "habitantes" de los túmulos, en toda la sierra nos encontramos con la también popular historia del héroe Roldán (Luengo Martínez 1952), gigante en muchas ocasiones, que por asuntos variados normalmente de amores, raptos, etc. (Carré Alvarellos 1977: 123-5) transita por esta región cruzándola en dos pasos de aproximadamente $1,5 \mathrm{~km}$. cada uno (otras versiones dicen que es tan solo el resultado de haberse bajado del caballo), dejando como es lógico 3 huellas de considerable tamaño en los siguientes lugares:

- Pena de Roldán en la aldea con dicho topónimo (Guitiri-Lugo).

- Ermita de San Vitorio, Galiñeiro (Guitiriz-Lugo), es la más conocida, en el límite provincial.

- Pena Ferreira, en el cordal del Vales (Aranga-Coruña).

Todas ellas en límites parroquiales y las dos últimas en límites municipales. Por otro lado todas coinciden con zonas de emplazamiento de túmulos ${ }^{15}$.

El "campesino" hace circular a Roldán por los lugares que son interpretados como factibles de paso dentro de la misma sociedad; esta leyen-

\footnotetext{
${ }^{15}$ En el caso de Pena de Roldán se trata del túmulo de A Forca Vella M 68 G 12 de los resultados de la prospección ya citada, situado al lado del arroyo de Porto Naveira. En Pena Ferreira disponemos referencias de su existencia.
}

"CUADERNOS DE ESTUDIOS GALLEGOS", Tomo XLI, Fascículo 106, Santiago 1993-94. 
da de Roldán no hace más que materializar una concepción espacial, explica el espacio tal y como lo define esa sociedad.

\subsection{Tercer nivel de análisis: arqueología del tránsito por Galiñeiro}

Si nos mantenemos en la escala general de la que hablabamos en el apartado 3.1., la primera característica que se aprecia inmediatamente en Galiñeiro es su función de:

- collado (como mínimo a la citada escala), permite una comunicación entre las dos zonas de valle una al $\mathrm{W}$ y otra al $\mathrm{E}$ del mismo. Esto considerando siempre la unidad de la sierra.

- ya indicamos además como en el $\mathrm{N}$ se da una solución de continuidad con el cordal de manera que surge la figura que expusimos como $\mathrm{cruz}$ (Cruceiro da Loba).

- para terminar en el S aparece el último tipo establecido, el porto, en lo que es paso del nacimiento del arroyo del Barranco da Loba hacia los campos de la aldea de Os Cainzos.

Todo ello armonizado de tal forma que el conjunto puede ser interpretado como una dorsal de estribación, atípica pero con los caracteres que para ella apuntabamos en 1.2.; con una solución de manual tanto la unión con las tierras altas (cruz conectando con corda) como con las bajas, pero con la curiosa cualidad de encontrar en su remate meridional otro bloque montañoso, de manera que el conjunto adquiere al mismo tiempo la definición ideal de collado que, por si fuera poco une dos sierras. Estamos pues ante un área de características extraordinarias como vía de tránsito que la convierten en un auténtico nudo en la canalización del movimiento de la región.

- tránsito en zonas altas.

- tránsito en zonas bajas.

- acceso en zonas altas.

- acceso de una zona baja a otra.

¿Por qué levantar tantos túmulos en la penillanura de Galiñeiro?

Opino que los constructores emplazaron allí sus tumbas porque les dió la gana, y esto no es una alegre sentencia a anatemizar sino un recordatorio de que tratamos con hombres, sociedades y culturas en los que, a este nivel, toda determinación natural por el hecho de ser asumida deja de ser tal para convertirse en algo que nosotros debemos intentar entender.

Veamos rápidamente los recursos que movilizaron los constructores de túmulos para construir. 


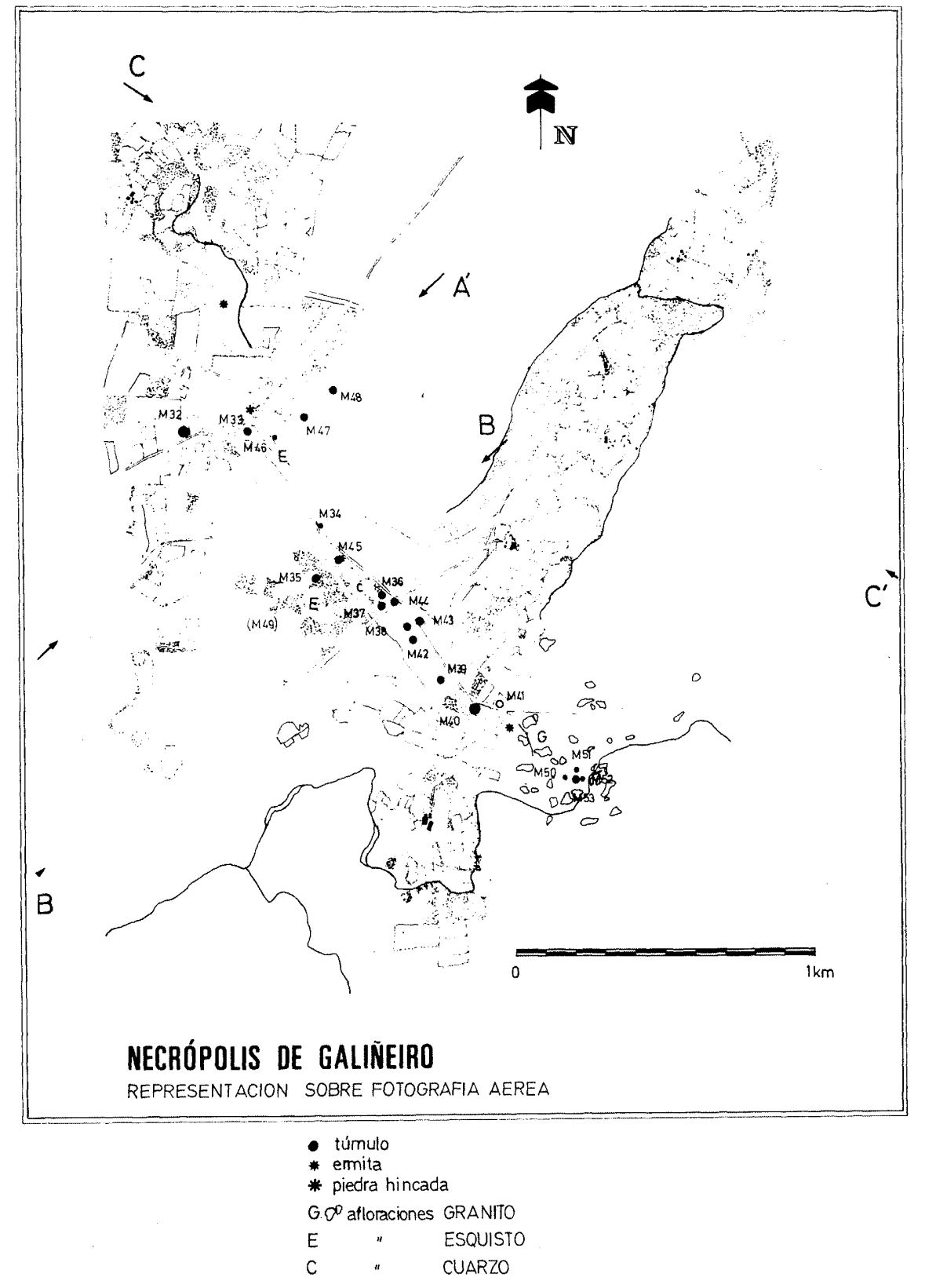

(c) Consejo Superior de Investigaciones Científicas 
(1) Túmulos y Geología. La situación del conjunto es la perfecta para hablar de la relación geología-megalitismo. Estamos ante un número elevado de arquitecturas en una superficie comparativamente pequeña, cuyo sustrato geológico presenta 4 tipos distintos de material, lo que ofrece la posibilidad de elegir a la hora de la construcción de la cámara. De nuevo las características geológicas vuelven a no determinar nada que valga la pena, tan solo una posible racionalización de lo que es la construcción, lo cual no nos parece nada novedoso si atendemos a la presunta no estupidez de los constructores. Si bien se da un predominio en el empleo del granito aparecen casos en los que éste no se utiliza (M 48) e incluso casos mixtos $(\mathrm{M} \mathrm{32})^{16}$. Tal y como proponíamos en el trabajo sobre A Reborica, observamos un simple aprovechamiento de los recursos disponibles; reconocemos (tal y como también decíamos) que el estudio de las diferencias de empleo de material puede ser interesante pero siempre que se parta de un marco no-determinante e inscribiéndolo en un análisis conjunto de la construcción, es decir, en todo caso un elemento más que contribuye a la creación de monumentalidad, una geología monumentalizadora.

(2) Túmulos y aprovechamientos. Observaciones en el plano. De nuevo la escala con la que trabajabamos en A Reborica vuelve a ofrecer resultados aquí, pudiéndose comprobar una serie de puntos al estudiar el aprovechamiento diferencial (Vaquero Lastres 1988) del espacio.

El conjunto tumular se emplaza en uno de los sectores que denominábamos de aprovechamiento extensivo frente a los que señalábamos como de ap. intensivo (labradío), de forma que parece vincularse otra vez y a través de esa especie de complejo situacional que podemos definir (Binford 1988) a las zonas de mejor disponibilidad agrícola que hoy en día son en donde se establece la población campesina, tanto las tierras inmediatas al collado por el E como los terrenos encajados por Galiñeiro - Cordal de Leira- Cordal de Vales en la parte occidental. Al igual que en el primero de los trabajos, si aislamos uno de esos sectores hallamos 2 yacimientos arqueológicos muy próximos; uno de ellos parece integrable en el grupo de los habitats "castreños" y otro, si bien la poca definición del recinto parece indicar también un núcleo de habitación, no vemos clara su adscripción cultural (¿quizás tiene que ver con los túmulos?). En todo caso vuelve a aparecer esa perduración en el uso y diferenciación de sectores en el entorno.

\footnotetext{
${ }^{16} \mathrm{~A}$ pesar de que los túmulos citados se sitúan a cierta distancia de la mancha granítica ésta sigue siendo escasa y permite plantearse una selección del material.
} 

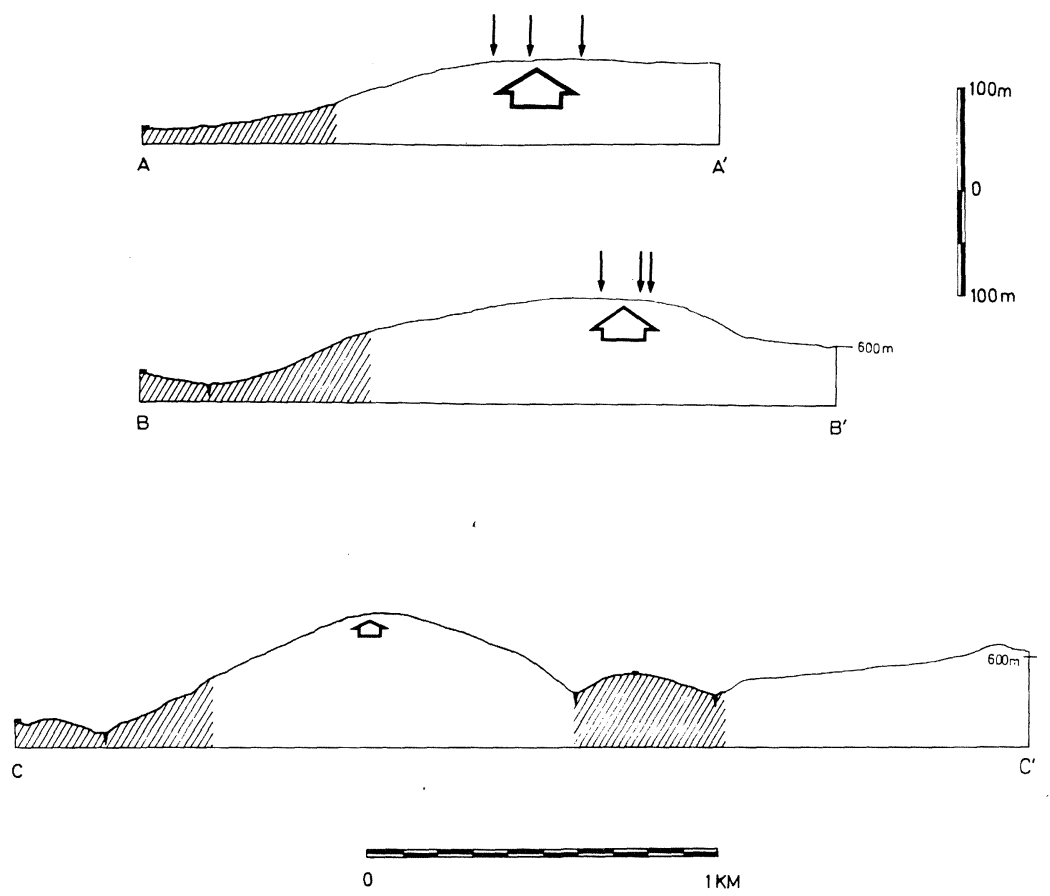

GALIÑNEIRO Secciones AA' BB' CC'

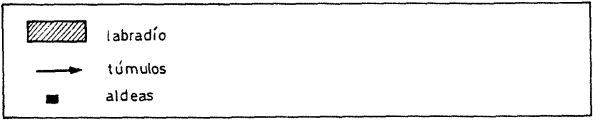

(3) Túmulos y visibilidad. Observaciones en el espacio. El estudio de la visibilidad nos habla también de esa relación entre los sectores arriba citados ya que la unidad fisiográfica queda perfectamente definida como conjunto por su visibilidad zonal incluso a varios kilómetros de distancia. Mientras que, por otro lado, arquitecturas concretas (visibilidad especifi$c a$ ) son divisibles desde zonas concretas y distancias dispares; desde el caso del M 32 destacado en el horizonte según accedemos a la cruz desde el W y probablemente desde el E (las alteraciones de la tumba no nos lo permiten comprobar), hasta la situación encajada del pequeño grupo de cuatro al SE de la ermita de S. Vitorio, volcados en el arroyo del Barranco da Loba, tan solo distinguibles desde un lugar cercano.

"CUADERNOS DE ESTUdiOS GALLEGOS", Tomo XLI, Fascículo 106, Santiago 1993-94. 

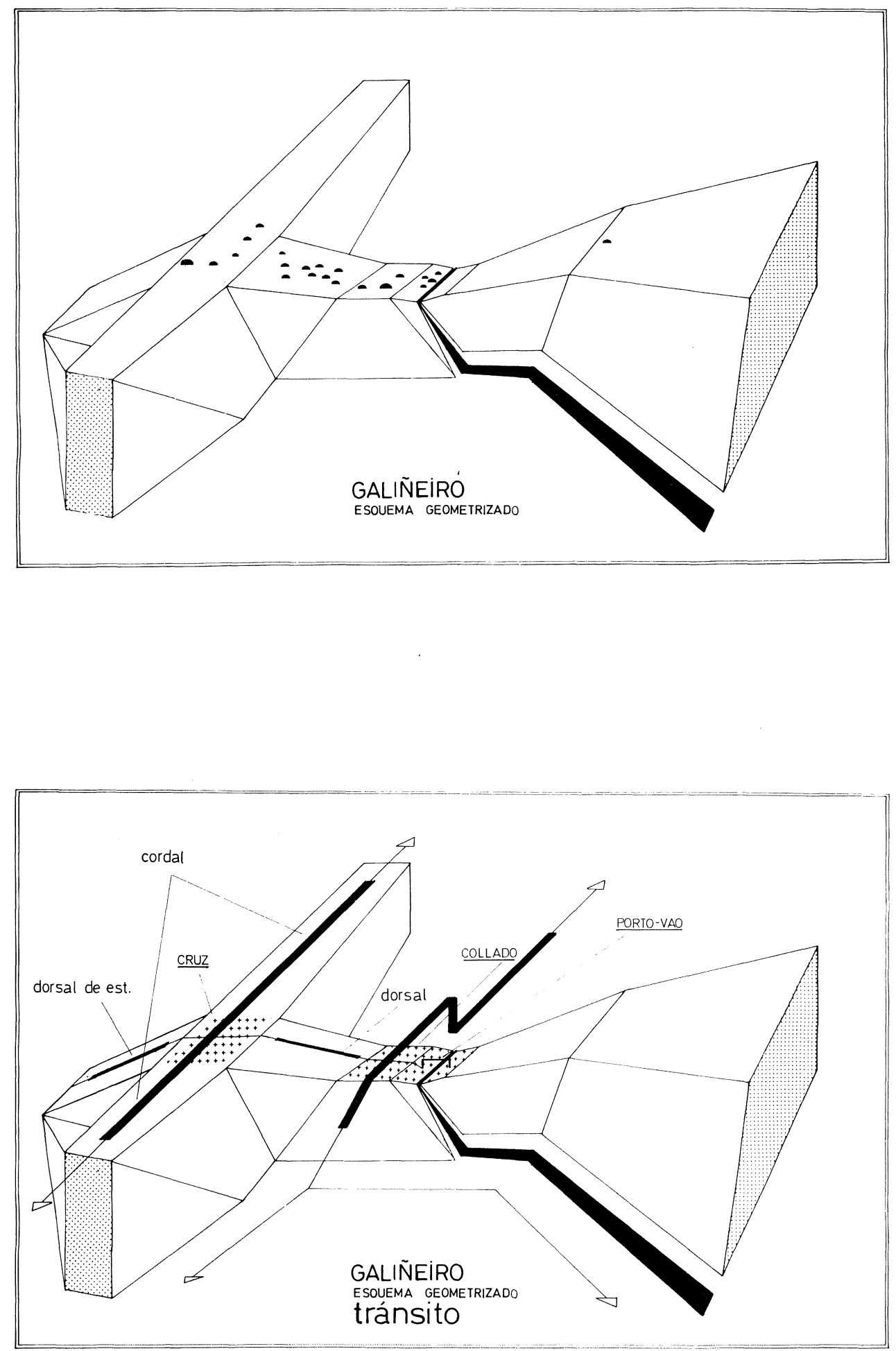

(c) Consejo Superior de Investigaciones Científicas 
Además de estos posibles recursos de monumentalización que la bibliografía sobre el tema en Galicia ya ha reconocido (Criado Boado A; 1988; 1989c; Criado Boado \& Vaquero Lastres 1990), podemos proponer ahora, y a la luz de los datos que este caso de estudio nos ofrece, que también la vinculación con un paso puede funcionar como factor de monumentalidad. Dos conjuntos de observaciones parecen bastante concluyentes en este sentido.

A.- Estamos ante un índice de concentración extraordinario como podemos ver en los gráficos siguientes ${ }^{17}$. Esto tal vez se deba entender teniendo en cuenta que GALIÑEIRO ES UN PASO:

Es un área que presenta en bloque unas características extraordinarias como vía de tránsito, aspecto que enlaza con los puntos arriba señalados. No es difícil darse cuenta de que es conveniente que un paso importante sea bien visible en su entorno a fin de poder utilizarlo, así como de que tampoco convendría que el lugar coincidiese con terrenos de labradío (aparte de condiciones geográficas distintas que suelen requerir).

En este punto debemos volver al caso de Roldán y su travesía por la región y comprobaremos como el campesino ha señalado con las huellas puntos clave en el tránsito del territorio. Así observamos que en Pena Ferreira no estamos más que ante un collado de sierra, lugar por donde se cruza el cordón de Vales, y el más confuso lugar de A Forca Vella no es otra cosa sino un porto de vadeo del arroyo Porto Naveira; y Galiñeiro, que disfruta de la huella más conocida y estimada, es también el más reseñado e importante paso.

B.- Tras habernos fijado en sus características arquitectónicas visibles, los tipos de las construcciones presentan importantes diferencias. Podemos encontrarnos diámetros de 35 a $10 \mathrm{~m}$. con alturas igualmente variables; la proporción túmulo-cámara tampoco se mantiene en los casos en los que se aprecia esta última; la coraza no es una constante; el propio material de construcción de la estructura interna; etc.

GALIÑEIRO COMO PASOS. Tenemos ya definido el conjunto como tal dentro de un paisaje tanto megalítico como natural. Ahora son las diferencias internas las que nos deben interesar ya que al igual que las anticipa la observación de las estructuras arquitectónicas, las vamos a detectar en la relación con las vías de tránsito.

\footnotetext{
${ }^{17}$ No olvidemos que esta apreciación la hacemos en función de los datos de una prospección rigurosa de una parcela representativa del espacio gallego tras una estudiada delimitación, no de una selección acomodada.
}

"CUADERNOS DE ESTUdios GALLEGOS", Tomo XLI, Fascículo 106, Santiago 1993-94. 
Hay dos ideas complementarias a destacar:

- En primer lugar, todos los accesos particulares a la Serra das Medoñas de Galiñeiro están vinculados a la visibilidad específica de algún túmulo (por supuesto estos accesos que son de muy distinto tipo), aunque esta no es una afirmación invertible, es decir, no todas las visibilidades específicas se relacionan con accesos a la sierra, algunas construcciones sólo son distinguibles desde posiciones internas de la unidad fisiográfica. Encontramos ejemplos como M 48 visibles desde la subida E, M 40 muy clara desde el S, o la propia M 32 perfectamente detectable desde el W; frente a otros que como M 36 solo se ve siguiendo el camino o ya dentro de la visualización de un grupo completo como también ocurre con alguno de los túmulos al S de la ermita de San Vitorio.

- En efecto, parece que existe una relación concreta de cada túmulo con los diferentes caracteres de paso que se dan en la zona de Galiñeiro y que ya definimos. Podemos ver el caso de M 32 que se vincula a un discurso en zonas altas, emplazada en una cruz-cordal con una visibilidad específica dominando un amplio territorio y accesos importantes (cuerda de A Loba y su extensión S, así como la subida desde el N); frente a otro muy distinto como es el del grupo de las (escondidas) vinculadas al vado del Barranco da Loba, paso del tipo porto cuya visibilidad específica quede bastante sumergida en el grupo y actúa en un espacio reducido y concreto. Por otro lado, casos como el citado M 12 totalmente insertadas en un grupo relacionado con la penillanura como tránsito de zonas bajas a altas (dorsal) o como collado en sí, de forma que su visibilidad depende de esa posición interna, de una ruta muy concreta y ¿quizás de un camino físico?

Queremos remarcar la importancia de la consideración del término porto dentro del análisis de emplazamiento y que creemos va a ayudar desterrar esa dudosa distinción que se venía haciendo entre megalitismo de montaña y de valle, explicando también esos considerados casos atípicos (monumentos ocultos al lado de los ríos, etc.).

\section{4.- CONSECUENCIAS}

Una agrupación de todas las ideas que hemos ofrecido concluye según nuestra opinión en 2 bloques de reflexiones en 2 ámbitos diferenciables.

"CUADERNOS DE ESTUdIOS GALLEGOS", Tomo XLI, Fascículo 106, Santiago 1993-94. 


\section{Consecuencias generales y perspectivas}

A la vista de nuestra experiencia de campo y conocimiento del fenómeno tumular gallego que ahora ejemplificamos en el conjunto de Galiñeiro, somos de la opinión de que las circunstancias que hemos detallado no hacen más que reflejar una vinculación de los túmulos a esas claves de tránsito en las dos vertientes que señalamos, proponemos dicha vinculación como el más uniforme de los factores de emplazamiento que nos encontramos en el NW, con la suma importancia que esto lleva implícito. De manera que creemos que la construcción va a estar siempre vinculada a una de esas áreas (tal y como las definimos arriba), lo cual, por supuesto, no implica que todas esas vías deban tener túmulos (de ahí nuestra preocupación por diferenciar el calificativo de natural en lo que se refiere a las vías).

En este sentido rechazamos toda relación apriorística con un camino físico pues no la consideramos extrapolable de modo general a nuestro tema de estudio.

Las ideas que fuimos desarrollando nos hacen pensar en una culturización de unas vías naturales de tránsito de manera que el registro arqueológico actual nos permite evidenciar la importancia, el uso de esa cruz de paso por las culturas constructoras de túmulos con lo que esto puede suponer en el conocimiento del movimiento de estas sociedades, utilidad que parece haberse mantenido el tiempo suficiente como para que se diese en la misma zona variaciones en los criterios de emplazamiento y construcción a una escala menor.

Y surge otra reflexión correlativa que anticipábamos al comienzo del artículo. En acuerdo con otros autores (Criado Boado 1989) vemos como esa culturización se hace a través de una actuación sobre la naturaleza, una artificiosidad al servicio de la ordenación del espacio ${ }^{18}$, una primera modificación de la naturaleza visible en el tiempo y en el espacio; en lugar de buscar los símbolos en la naturaleza directamente, se van a crear esos símbolos activos que son los monumentos tumulares. ¿Es este el caso de los caminos? ¿son alteraciones simultáneas del mismo tipo?; creemos que esto no fue así y tan solo dejamos una posibilidad en las etapas más tardías de la necrópolis.

${ }^{18}$ Dentro de este proceso es donde habría que enmarcar todo lo dicho a la oposición zona de vivos - zona de muertos (Vaquero Lastres 1988).

"CUADERNOS DE ESTUdiOS GALLEGOS", Tomo XLI, Fascículo 106, Santiago 1993-94. 
Estamos de nuevo, por otro lado, en lo que es la posible construcción monumental que aquí no solo se descubre en un elemento concreto, en una obra, sino también de un espacio unitario ${ }^{19}$.

\section{Consecuencias particulares: interpretaciones}

¿Cómo continuar? es bien simple. La relación túmulos-vías de tránsito nos acerca a la idea de un emplazamiento quizás monumentalizante de forma que éste podría pasar a ser estudiado inserto en el resto de elementos ya definidos (arquitectura y condiciones, restos interiores, etc.).

Hacer alguna propuesta interpretativa en el estado que hemos dejado al investigador resulta muy aventurado ${ }^{20}$ pero si podemos tener en mente alguna idea.

La visibilidad se relaciona con el camino.

El camino con el movimiento de las comunidades.

Las prioridades del tránsito con la periodización cronológica dentro de la necrópolis.

Del mismo modo que pudimos definir en la Serra das Medoñas de Galiñeiro un carácter claro de lugar de tránsito en diferentes áreas y de forma diacrónica, para luego temporalizar el conjunto mediante la constatación de una cultura material definible, podemos dirigirnos a la temporalización relativa de elementos concretos en virtud de su relación con las vías. Pretendemos abrir una puerta al estudio del movimiento de las sociedades constructoras de túmulos.

Santiago en primavera de $1989^{*}$

\footnotetext{
${ }^{19}$ Descubrimos una vez más la diferencia de escalas que llevamos manteniendo en todo el discurso y que es la que nos legitima a utilizar repetidas veces la palabra necrópolis.

${ }^{20}$ Para ello deberíamos trabajar con una escala que comprendiese a todo el terreno prospectado o en su defecto llevar a cabo un análisis de todos los elementos incluyendo datos de excavación. Ni que decir tiene que lo ideal sería una conjunción de ambas posiciones.

* Este texto ha sido referido a veces por mí o algún compañero como trabajo en prensa en esta misma revista. Fue depositado para su publicación en el TOMO XL 105, junto con una aplicación puntual derivada de las ideas aquí expuestas. Dicha consecuencia práctica escrita con la colaboración de CRIADO BOADO, con quién estudié los túmulos del Bocelo, y INFANTE ROURA, transcriptor del diario de seguimiento de un grupo de caballos, apareció en el volumen carente por lo tanto los fundamentos que lo motivaron (apartado 3.2 de este texto). Este es el motivo de la aparición pues de este trabajo antiguo pero continuo, juzgando interesante a pesar de que su temática la he desarrollado en otros artículos.
}

"CUADERNOS DE ESTUDIOS GALLEGOS", Tomo XLI, Fascículo 106, Santiago 1993-94. 


\section{BIBLIOGRAFÍA CITADA}

Alsina, J., 1986. Etología, ciencia actual. Barcelona: Anthropos; 153 pp.

Bello Diéguez, J.M., 1988. Informe sobre los trabajos arqueológicos llevados a cabo en el dolmen de Dombate y la Cha de Borneiro. Depositado en los Servicios de Arqueología de la Xunta de Galicia.

Bello Diéguez, J.M., Criado Boado, F. \& Vázquez Varela, J.M. 1982. Sobre la cultura megalítica y los caminos antiguos en Galicia. Revista del Museo de Pontevedra, tomo XXXVI; pp. 1-21.

Bello Diéguez, J.M. Criado Boado, F. \& Vázquez Varela, J.M. 1987. La cultura megalítica de la provincia de La Coruña y sus relaciones con el marco natural: implicaciones socio-económicas. Coruña: Diputación Provincial; 163 pp.

Binford, L., 1988. En busca del pasado. Descifrand el registro, arqueológico. Barcelona: E. Crítica (London: Thames \& Hudson, 1983); 283 pp.

Carre Alvarellos, L., 1977. Las leyendas tradicionales gallegas. Madrid: Espasa-Calpe, col. Austral; 303 pp.

Coles, J.M., 1990. La carrera más antigua del mundo, Investigación y Ciencia 160. pp. 70-77.

Criado Boado, F., 1981. Relación entre la distribución de mámoas y el medio físico en la zona de Sobrado-Curtis. Brigantium 2.

Criado Boado, F., 1984. Prospecciones de túmulos megalíticos en la provincia de A Coruña-Campaña del 84: A serra do Bocelo. Santiago: Xunta de Galicia, Dirección Xeral do Patrimonio Histórico e Documental.

Criado Boado, F., A. Contribución al estudio de las relaciones entre las comunidades megalíticas del NW peninsular y su medio natural: implicaciones socio-económicas. Universidad de Santiago, tésis doctoral presentada en la Facultad de Xeografía e Historia. 
Criado Boado, F. \& Fábregas Valcarce, R., 1989a. The megalithic phenomenon of northwest Spain: main trends. Antiquity vol. 63, núm. 241; pp. 682-96.

Eibl-Eibesfeldt, I., 1983. El hombre preprogramado. Lo hereditario como factor determinante en el comportamiento humano. Madrid: Alianza núm. 176 (Wien-München-Zürich: Verlag Fritz Molden, 1973); 325 pp.

Filgueira Valverde, J. \& García Alen, A., 1977. Inventario de monumentos megalíticos de la provincia de Pontevedra. El Museo de Pontevedra, XXXI; 82 pp.

Grupo de Arqueología "Federico Maciñeira" (E.N.D.E.S.A.), a. Trabajo de catalogación de monumentos tumulares en la comarca de As Pontes en base a estudios de los propios miembros y de Federico Maciñeira.

Hernández San Pelayo, 1922-42. Hierros de Galicia. Memórias del Instituto Geológico y Minero de España, tomo III, cap. XI. Madrid.

Jorge, V.O., 1984. Megalitismo no Norte de Portugal: novos elementos. Revista de Guimaraes 94; pp. 263-289.

Lorenz, K., 1986. Fundamentos de la etología. Estudio comparado de las conductas. Barcelona: Paidos (New York: Springer-Verlag/Wien, 1978); $349 \mathrm{pp}$.

Luengo Martínez, J.M., 1952. Exploraciones de mámoas de Aranga y Cambás. Cuadernos de Estudos Galegos, tomo VII, fasc. 22.

Maciñeira y Pardo de Lama, F., 1929. Notable grupo de círculos líticos y túmulos dolménicos de la cuenca superior del Eume. Arquivo do Seminario de Estudos Galegos II; pp. 197-230.

Maciñeira y Pardo de Lama, F., 1941. El vaso campaniforme y condiciones de sus intactos yacimientos tumulares en la estación de Puentes, del cabo Ortegal. Atlantis XVI; pp. 356-369. 
Maciñeira y Pardo de Lama, F., 1943-44. Túmulos prehistóricos. Inventario descriptivo de los 286 túmulos prehistóricos hasta ahora descubiertos en la avanzada comarca del cabo Ortegal. Boletín de la Real Academia Gallega XXIII-XXIV.

Maciñeira y Pardo de Lama, F., 1943. Bares. Puerto hispánico de la primitiva navegación occidental. C.S.I.C.; capit. I y IV.

Maciñeira y Pardo de Lama, F., 1935. La distribución de las estaciones prehistóricas ortegalesas y sus características. Boletín de la Real Academia Gallega XXII.

Patiño Gómez, R., 1985-86. La cultura megalítica en el valle del Fragoso. Pontevedra arqueológica II; pp. 17-34.

Patiño Gómez, R. \& García Lastra Merino, M., 1987. Tres conjuntos megalíticos de la provincia de Pontevedra. Gallaecia 9/10; p. 211-220.

Pérez Outeiriño, B., 1978. A necrópolis megalítica de A Moura. Boletín Auriense VIII; pp. 315-321.

Pombo Mosquera, X.A., 1986. O megalitismo no NW da Terra Cha (Lugo): relación de o home e o medio. Gallaecia 7/8; pp. 225-231.

Rodríguez Colmenero, A., 1970. La cultura megalítica en el Alto Budal. Boletín Auriense, año I, tomo I; pp. 31-60.

Sobrino Lorenzo-Rouza, R., 1953. Megalitos del Monte Corzán. Zephyrus IV; pp. 177-186.

Vaquero Lastres, J., 1988. Prospección en Serra da Loba y Cordal de Montouto: el fenómeno tumular. Santiago: Xunta de Galicia, Dirección Xeral do Patrimonio Histórico; memoria inédita, (una versión de este traballo aparecerá publicada en Brigantium).

Vaquero Lastres, J., 1989. ¿Donde diablos se esconden nuestros muertos que no los podemos ver? Reflexiones sobre el emplazamiento de los túmulos del NW. Gallaecia 11; pp. 81-108. 
Vaquero Lastres, J.A. Excavación en los túmulos 165 y 166 de O grou en As Pontes de García Rodríguez. Memoria inédita.

Vázquez Seijas, M., 1953. Notas sobre túmulos lucences. Zephyrus $I V$; pp. 219-225. 